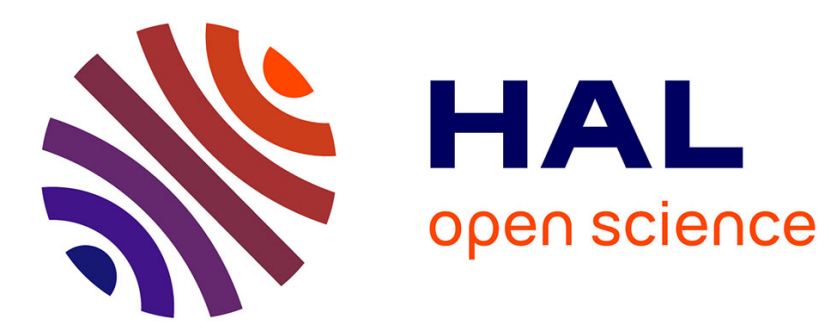

\title{
La grande statuette en bronze de Kerguilly-en-Dinéault (Finistère) \\ René Sanquer
}

\section{To cite this version:}

René Sanquer. La grande statuette en bronze de Kerguilly-en-Dinéault (Finistère). Gallia - Fouilles et monuments archéologiques en France métropolitaine, 1973, 31 (1), pp.61-80. 10.3406/galia.1973.2625 . hal-01934109

\section{HAL Id: hal-01934109 \\ https://hal.science/hal-01934109}

Submitted on 11 Mar 2020

HAL is a multi-disciplinary open access archive for the deposit and dissemination of scientific research documents, whether they are published or not. The documents may come from teaching and research institutions in France or abroad, or from public or private research centers.
L'archive ouverte pluridisciplinaire HAL, est destinée au dépôt et à la diffusion de documents scientifiques de niveau recherche, publiés ou non, émanant des établissements d'enseignement et de recherche français ou étrangers, des laboratoires publics ou privés.

\section{(이) $\$$}

Distributed under a Creative Commons Attribution - NonCommercial - NoDerivatives $\mid 4.0$ 


\title{
LA GRANDE STATUETTE DE BRONZE DE KERGUILLY EN DINEAULT (Finistère)
}

\author{
par René SANQUER
}

Dans le domaine de la sculpture, en pierre ou en métal, l'Armorique romaine a rarement livré, jusqu'ici, des œuvres de très grande qualité1. Aussi la présence, à l'extrême Occident du monde antique, d'une statue exprimant avec autant de bonheur les grandes composantes de l'art européen peut sembler surprenante à bien des égards (fig. 1). Il aura fallu, pourtant, plus d'un demi-siècle après sa découverte pour que son existence soit connue et son importance, proclamée.

Au mois de mai 1913, en effet, II. Jean Labat, jeune cultivateur demeurant au hameau de Kerguilly en Dinéault ${ }^{2}$, au cours d'un labour profond dans une lande nommée Gorré-ar-C'hoad (le haut du bois), nouvellement défrichée et jamais cultivée de mémoire d'homme, recueillit, sous sa herse, une petite tête de bronze en très bon état de conservation, éraflée seulement à la pointe du nez et sous l'œil gauche par le soc de la charrue. Cette lande occupe le sommet d'un mamelon aux flancs abrupts, séparé du versant oriental du .Yenez-Hom par une profonde vallée, à $3,200 \mathrm{~km}$ de son sommet ${ }^{3}$. Après un intervalle de plusieurs années, dû à la guerre et à l'oubli, J. Labat entreprit de vérifier, vers 1928 , si le reste de la statue n'était pas demeuré en terre. Il découvrit une cavité cylindrique, creusée dans la glaise. Large de $50 \mathrm{~cm}$, profonde de $1 \mathrm{~m}$, elle était soigneusement aménagée, les bords lissés, le fond en forme de cuvette et, semble-t-il, recouverte d'une galette d'argile. A l'intérieur se dressait, debout, un corps féminin en bronze, revêtu d'une longue robe à plis, sans ceinture, avec, selon le témoignage de J. Labat, "des épaulettes et, sur la poitrine, une sorte de collier de la Légion d'honneur $"^{4}$. Deux bras nus, encore en place, et des pieds chaussés

1 En dehors des petites statuettes de bronze et de terre cuite, citons néanmoins la grande statuette d'Hercule, provenant de Plomare'h à Dournenez (Finistère), Espérandiec, Recueil général des bas-reliefs, stalues el bustes de la Gaule romaine, Paris, à partir de 1907, no 7168 et Gallia, XV, 1957, p. 188 ; la tête de divinité féminine de Crach (Morbihan), Espéraxilst, op. cil., no 302-9, les deux têtes féminines d'époque sévérienne, en marbre blanc, provenant de Port-de-Roche en Langon, canton de Jedon (Ille-et-Vilaine), voir Bull. de la Soc. arch. d'Ille-et-Vilaine, 1929, p. xı.

2 Canton de Chateaulin (Finistère). Je tiens de .I. Jean Labat, lui-même, les renseignements sur les circonstances de la découverte. Ses propos ont été enregistrés au magnétophone et sont conservès à la Faculté des Lettres de Brest. Pour plus de détails, cf. R. Sanguer et D. Lalrevit, La déesse cellique du Menez-Hom, dans Bull. de la Soc. arch. du Finistere, XCVII, 1971, p. 85-108. Que J. Labat veuille bien trouver ici l'expression de mes remerciements.

3 Coordonnées Lambert : 79, $3 \times-115,5 \mathrm{E}$; coordonnées géographiques : 4${ }^{\circ} 12^{\prime} \mathrm{O}, 48^{\circ} 13^{\prime} \mathrm{N}$.

4 J. Labat est chevalier de la Légion d'honneur à titre militaire. Il compare, sans doute, l'égide à la cravate de commandeur de la Légion d'honneur. 


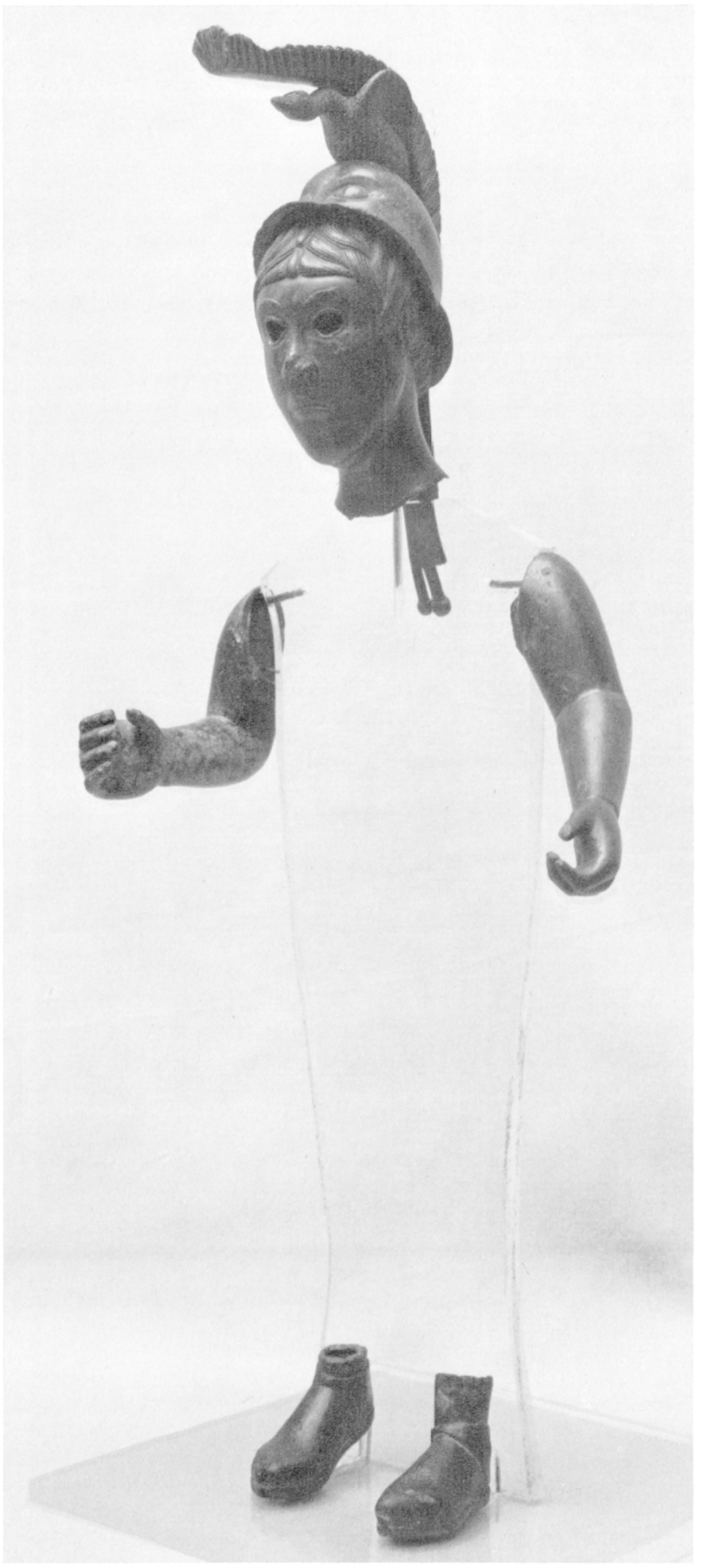

1 Éléments de la grande statuette de bronze de I)inéault. Hant. approximative: $0,70 \mathrm{~m}$ 
de sandales, complétaient l'ensemble. Au fond de la cavilé, l'inventeur recueillit un casque, un oiseau et un cimier brisé. I a statue, au total, aurail mesuré 0,70 m environ. Nalheureusement, le corps était en trìs maurais état, la tòle de bronze martelé qui te constituait, "épaisse comme une feuille de papier à cigarettes", ayant été profondément altaquée par la corrosion. Au moment de la découverte, la partie antérieure du corps formait encore une seule coque, mais l'arrière n'élait déjà plus qu'une dentelle informe qui, entreposée dans un local humide, disparut rapidement. Les autres pièces, en bronze fondu, plus épais, recouvertes d'une paline vert foncé avaient conservé une qualité parfaite. Elles furent données par J. Labat à son médecin, le Ior Vourch, en mème temps que la partie antérieure du corps, vers 1935. Pendant la seconde guerre mondiale, les éléments de la statue furent enfouis a nouveau, pour échapper aux occupants allemands, et exhumés vers 1945. Le devant du corps était si endommagé que l'on ne jugea pas utile de le récupérer. Les pièces sont restées dans la famille du Jor Vourc'h jusqu'en 1972. Elles appartiennent aujourd'hui aux collections du Ilusée de Rennes.

Quelques observations sur le cadre géographique el historique peuvent aider à replacer la stal ue dans son contexte. Le Menez-Ilom n'est pas une colline quelconque. Arec ses $330 \mathrm{~m}$ d'altitude, il fait figure de relief important et mérite, en breton, le nom de montagne (menez). A $6 \mathrm{~km}$ seulement de la mer, son sommet, dénudé aujourd'hui, se découpe à l'horizon du navigateur qui aborde soit le soulet de Brest, soit la baie de Douarnenez (lig. 2). Si l'étymologie du second terme, Hom, n'est pas (lairement établie (rertains y voient le nom de saint Crome), la présence, près du sommet, d'une chapelle placée sous le vocable de sainte Marie laisse penser que la montagne a été dédiée de longue date a la Vierore chrélienne. Au pied du IIenez-Hom, vers le sud-ouest, le Porzay, petit "pays" bien individualisé, a été le siège, dans l'Antiquité, d'une importante industrie du garum et des salaisons qui justifie le grand nombre de vestiges romains bordant la baie de Douarnenez et la prospérilé de cette région pendint les quatre premiers siècles de notre ère ${ }^{5}$. Cette richesse explique de mềne le groupement, dans un espace restreint, de trois temples celto-romains, les seuls connus, jusqu'ici, dans la vaste cité des Osismii. Le temple de la presqu'île de Crozon est assez mal localisé6 mais l'emplacement de celui de Plomodiern a été retrouvé récemmenti . Le troisième fanum, à Tregouzel en Douarnenez, a été fouillé plus complètement : on y a relevé deux niveaux bien distincts, l'un, de l'époque d'luguste, l'autre, du ${ }_{1}^{\mathrm{e}}$ siècle de notre ère ${ }^{8}$. On y trouve aujourd'hui, en surface, de la céramique sigillée d'Argonne. La commune de IJinéault ne fait cependant pas partie du Porzay. Elle s'avanre, sur le versant nord-est, jusqu'au sommet du Venez-Hom et son territoire est contigu à celui de la commune de Plomodiern. Aucune trace de l'occupation romaine n'y a jamais été signalée. Par contre, la présence gauloise y est marquée par trois souterrains-refuges 9 . Une très ancienne piste,

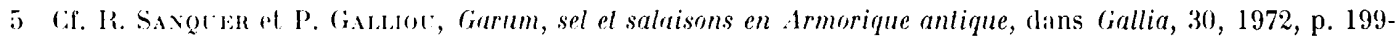
2:23. Voir notamment, p. :201, une carte des vestiges gallo-romains de la haie de Ioouarnene\%.

6 L'arehéologur qui a fait connaître ce lemple et son plan indique seulement qu'il se trouve vis-à-vis l'extrémité n. de la bair de Douarnenez ot en vue de son point de jonction ave l'occin, pres de Crozon. On est donc fonde à le siture pris du cap de la Chevre lif. Ins

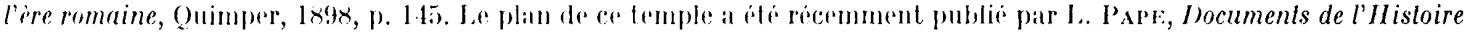
de la Brelatgne, l'.1rmorique gallo-romaine, Toulouse, 1971 , p. 5i).

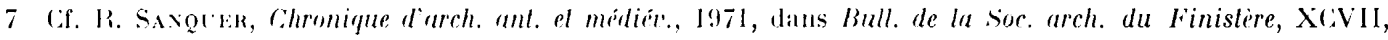
1971 , p. 19-50. Voir le plan dans 1. Paps, oner. cile, p. 52. Halna du Fretay fo situe "en vue de la mer, baie de

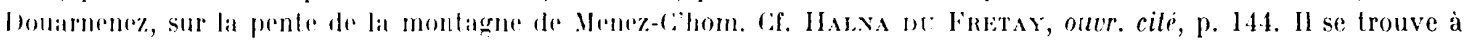

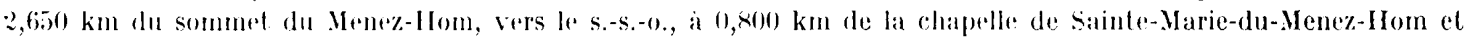

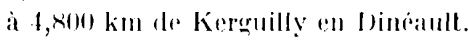

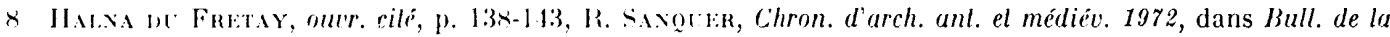
soc. arch. du Finistire, Navilu, 1972, p. 1x.

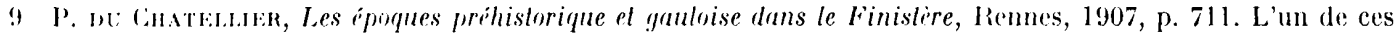
souterrains, an moins, a livri de la ciramique ganloise. lls figurent tous les trois sur la carte de repartition des souterrains armoricains de l'Ige du Fer, par P.-R. Gor, Documents de l'Hisloire de la Brelayne, Préhisloire el protohistoire armoricaines, Toulouse, 1971, p. 39. 

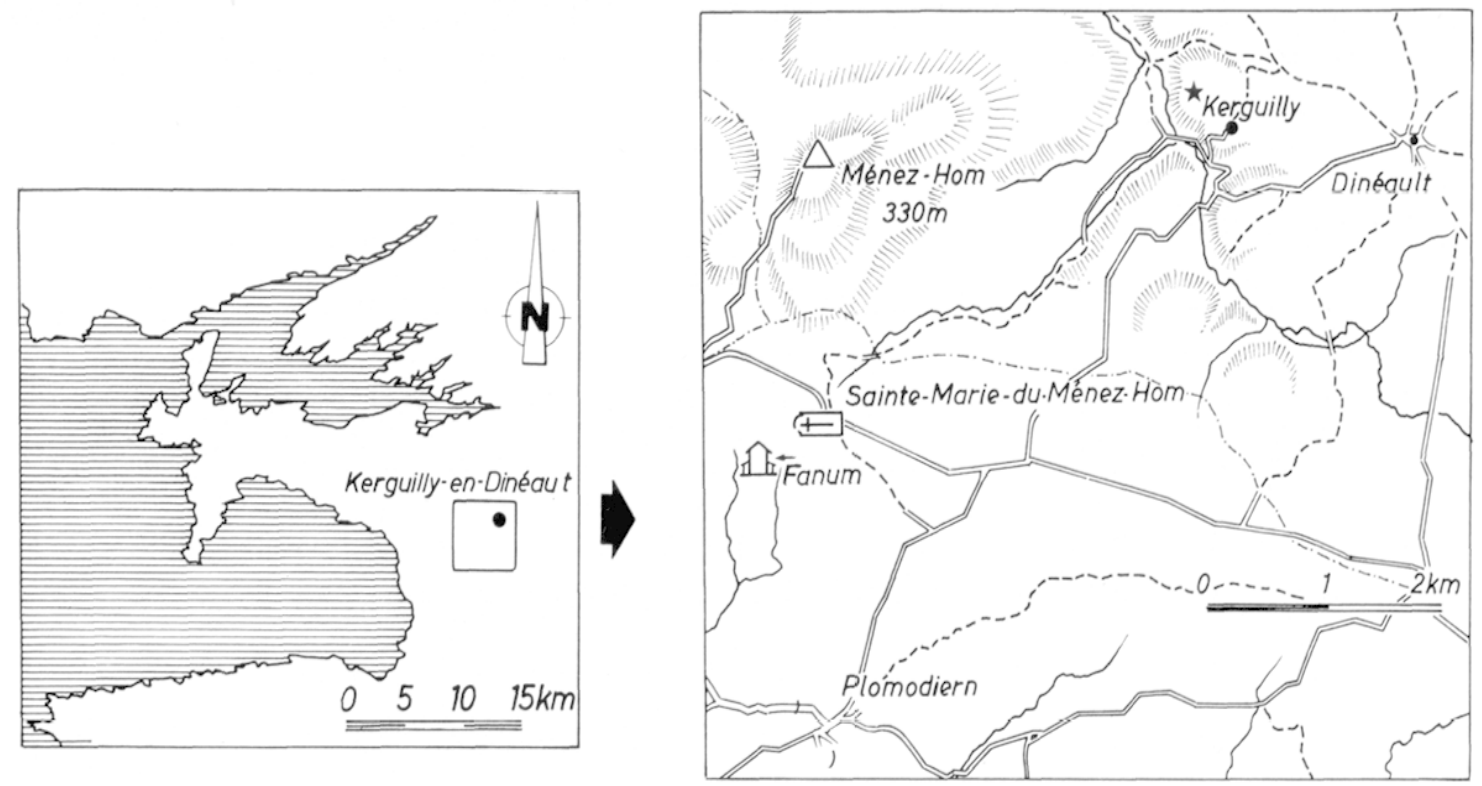

2 Localisation de la trouvaille de Kerguilly en Dinéault.

desservant la presqu'île de Crozon, passe à proximité du bourg. Le champ lui-même, où la statue a été découverte, n'offre, aujourd'hui, aucun indice particulier. Le site semble propice à l'établissement d'un habitat fortifié et, il y a quelques années, J. Labat y a détruit un rempart de terre mesurant $100 \mathrm{~m}$ de long, $2 \mathrm{~m}$ de large et $2 \mathrm{~m}$ de haut, parementé à l'extérieur de pierres sèches et percé d'une porte également parementée. Une photographie aérienne prise en 1948 montre les restes d'une enceinte quadrangulaire ${ }^{10}$. C'est au pied du rempart, vers l'intérieur, que la cachette avait été creusée. Ce mode de protection du matériel religieux n'est pas exceptionnel : d'autres caches similaires, renfermant des statues ou des objets de culte, ont déjà été découvertes dans le passé, souvent à proximité de sanctuaires ${ }^{11}$, et la trouvaille de Neuvy-en-Sullias (Loiret) provient d'une fosse de ce genre, quoique, semble-t-il, mieux protégée ${ }^{12}$. Il n'est donc pas trop aventureux d'avancer l'hypothèse que la statue de Kerguilly, placée à l'origine dans un sanctuaire public ou privé, ait été cachée là intentionnellement, à l'approche de quelque danger. Ce sanctuaire était-il le fanum celtoromain de Plomodiern ou le laraire de l'habitat fortifié de Kerguilly? Il est impossible de se prononcer.

L'objet lui-même, d'une hauteur restituée approximativement de $0,70 \mathrm{~m}$, appartient, par la technique et le style, à une série de statues de bronze de petite taille, ou de grandes statuettes, que l'on retrouve dans les provinces périphériques de l'Empire romain, mais avec une plus grande fréquence dans l'ancien domaine celtique ${ }^{13}$. Tous les éléments de

10 Photographie IGN, mission 6, GR 1/33 du 16-IV-1948, nos 62-63.

11 F. Brafmer, Sculplures en métal batlu el repoussé de la Gaule romaine et des régions limilrophes, dans Revue archéologique, 1969, 1, p. 101, cite le fait que la plupart des grandes statuettes de métal ont été mises à l'abri dans des cachettes aménagées à proximité de sanctuaires de montagne ou de plaine.

12 Pour le trésor de Neuvy-en-Sullias, voir P. Maxteli.ier, Mémoires sur les bronzes antiques de Neuvy-enSullias, dans Mémoires de la Soc. arch. de l'Orléanais, IX, 1866, p. 171-173. Le trésor de Marengo (Alessandria, Italie) a été découvert dans une cachette creusée dans le sol, cf. G. Brexdicels, Il tesoro di argenteria di Marengo, dans Monumenti d'arte antica editi della Reale Accademia delle Scienze di Torino, fasc. 1, Turin, 1937, p. 3.

13 On a retrouvé en Dacie un tout petit masque de bronze représentant la déesse Bendis (?) et offrant une certaine similitude de facture et de style avec les bronzes de la zone celtique, cf. The Illyrians and Dacians, Belgrade. 1971, no D 234, notice de H. Daicoviciu. Pour les bronzes de Gaule et d'Angleterre, voir infra. 
la statue aujourd'hui conservés sont en bronze coulé, à l'exception d'une douille de bronze martelé enfoncée dans la chaussure grauche. Nous n’entrerons pas dans le détail des procédés de fonte, bien connus des spécialistes et, par ailleurs, fort complexes. Ici, la technique de la fonle creuse a été utilisée et des fragments de noyau ont été préservés à l'intérieur des bras et de l'oiseau. L'analyse a montré que l'artisan s'était servi d'un sable de fonderie, riche en fer ${ }^{14}$. Sur le côté de la tète, derrière l'oreille et légèrement au-dessus, une mince bande de tôle de bronze a été insérée, pour réparer, sans doute, un défaut d'exécution au moment du démoulage (fig. 4 , c). La patine, d'un vert plus clair, indique une composition différente, que nous n'avons pas pu analyser faute de pouvoir prélever un échantillon. Aucune trace de soudure n'apparaît aux articulations. A la base du cou, une feuillure d'attente, en retrait de 4 à $5 \mathrm{~mm}$, visible sur tout le pourtour, et haute de 6 à $7 \mathrm{~mm}$, est prévue pour être insérée à force dans un orifice plus étroit. Lne feuillure identique permettait aux bras de s'encastrer de la même manière dans les épaules. Quant aux sandales, l'une, à gauche, porte, nous l'avons dit, une partie moulée, l'autre, à droite, est munie d'un collier d'attente (fig. 8). Le casque, l'oiseau, le cimier, devaient ètre maintenus solidaires par une goupille verticale. In trou circulaire perce en effet chaque objet et permet de les réunir. L'axe, cependant, a disparu, et on n'en connaît pas la matière : il s'était anciennement rompu et on s'explique que J. Labat ait retrouvé les pièces au fond de la cavité. Le casque s'adaptait sur la tête à l'emplacement de la calotte crânienne. Une bande lisse, large de 3 à $4 \mathrm{~mm}$, prolonge directement la chevelure, sans le retrait caractéristique de la feuillure du cou et des bras (fig. 4, b, c, d). Cette lèvre d'attente facilitait la fixation du casque. L'absence de boîte crânienne apparente notre statue, sur le plan technique au moins, à une série d'œuvres d'art retrouvées sur le territoire de l'ancienne Gaule ${ }^{15}$. Dans la plupart des cas, le sommet du crâne est absent, la chevelure est brusquement interrompue et une feuillure d'attente permet la fixation d'un couvre-chef ou d'un couvercle. La raison d'ètre de cette disposition, qui ne semble pas due à une raison technique, reste obscure. Pour la statuette de Dinéault, cependant, la présence du casque et de ses appendices constitue une explication suffisante.

Par la technique générale de fabrication, la statue de I)inéault, se rapproche plutôt de colle du dieu bien connu de Bouray ${ }^{16}$. En effet, dans ce dernier cas, le corps est fait de tôle de cuivre, en deux coques; la tête, en fonte creuse, est formée de deux éléments, l'un constituant le visage et la partie antérieure du crâne, l'autre, l'occiput et la nuque. Il ne faut cependant pas cacher les différences : l'ajustage des pièces du dieu de Bouray est réalisé par la soudure au plomb de plaques juxtaposées, les joints sont recouverts

1.t Voir infra, p. 67, le tableau des analyses du métal et du sable.

15) Votamment un objet au repoussé, le Jercure de Berthouville, cf. F. BRAFmer, art. cilé, 1968, 2, p. 351-35.4, fig. 3.4-3x, et trois aeuvres moulees, le Mercure de Thiennes CNord, cf. S. Botchen, Trois bronzes divins de Thiennes (Nord), dans Ciallia, 30, 1972, p. 127-148; la grande statue de Coligny, cf. J. Bccrif, Le Mars de Coligny (.Musée de Lyon), dans Monuments... P'iot, X, 1903, p. 70 ; le buste de Dogmart-en-Ponthieu (Somme), conservé aul llusée du Iouvre, ef. A. DE: Rmble, Les bronzes antiques du Louvre; I. Les figurines, Paris, 1913, p. 14, no 47. II me s. Boucher art. cité, p. 135; fait remarquer que, dans le cas d'un bronze moulé, rien n'est plus facile pour un artisle habile que de réaliser en même temps la tête et la coiffure.

16 K. Laxtra, Le Dieu celtique de Bouray, dans Monuments... Piot, XXXIV, 1934, p. 35-j8; I. Braknkr, art. cilé, p. 86-88. 

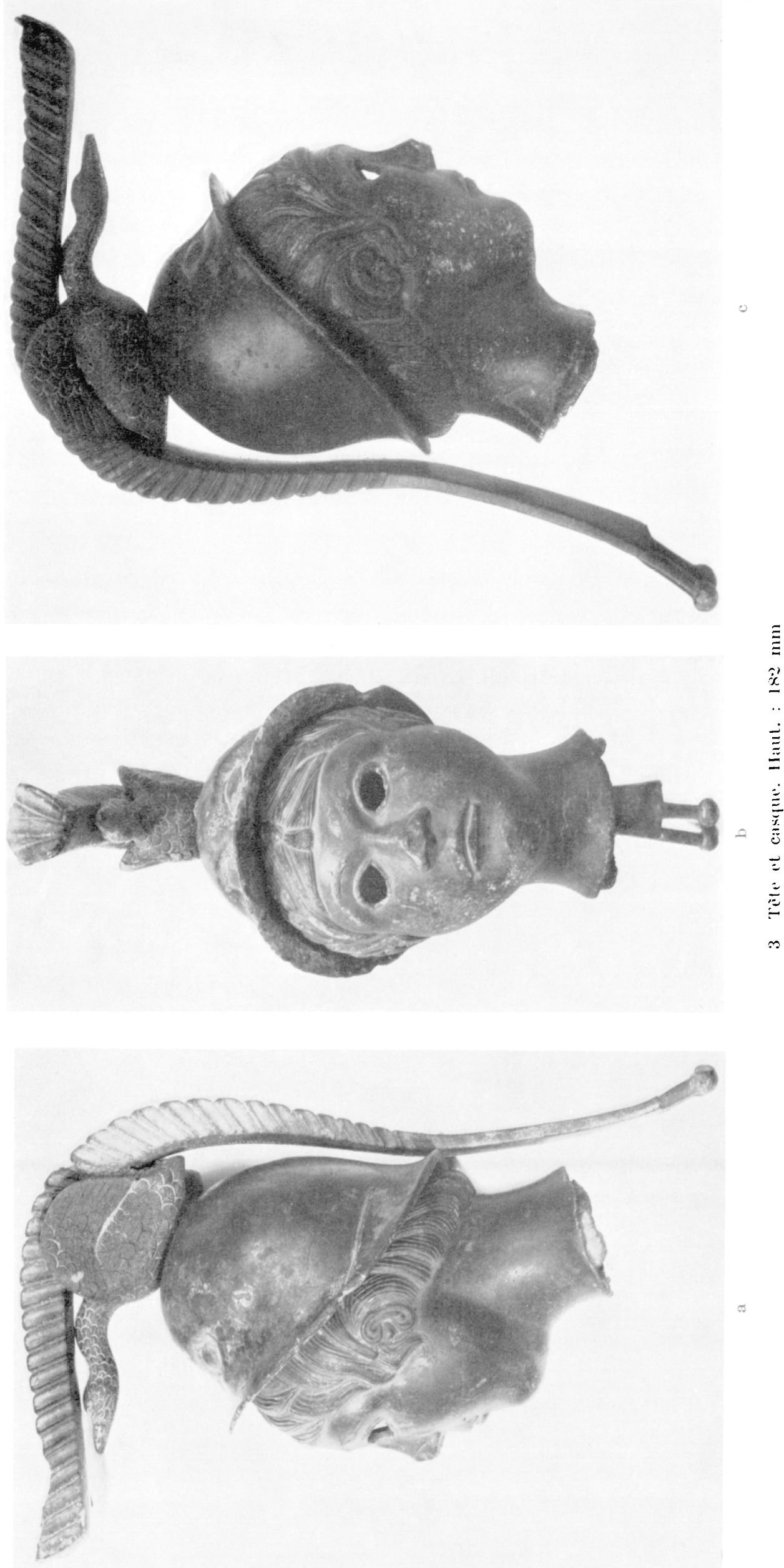
de baguettes de cuivre, et la division de la tête en deux parties évoque plutôt les chefs reliquaires du Moyen Age.

Ainsi done, si l'on s'en tient à la technique seule, la statue de Jinéault n'est pas étrangère à la production gauloise, tant d'œuvres de goût classique que d'œuvres plus proches des sources indigènes. C'est, d'ailleurs, ce que montre également l'analyse de la 'omposition du métal, dont les résultats sont reproduits ci-dessous. La tête, le casque, les bras, les chaussures, sont faits d'un alliage tornaire de cuivre, d'etain, de plomb. L'irrégularité des coulées, les procédés empiriques de fabrication, ne permettent pas de comparaisons très strictes, mais la proportion de cuivre avoisine les $7.5 \%$, celle de l'étain $13 \%$, celle du plomb $8 \%$, ce qui correspond aux teneurs relevés pour d'autres bronzes romains ${ }^{17}$. Le zinc est presque partout présent. L'intérieur de la chaussure droite a ćté rempli d'un alliage de plomb et d'étain pour assurer une bonne stabilité.

AXATYE DE TA COMPOSITION JU META.

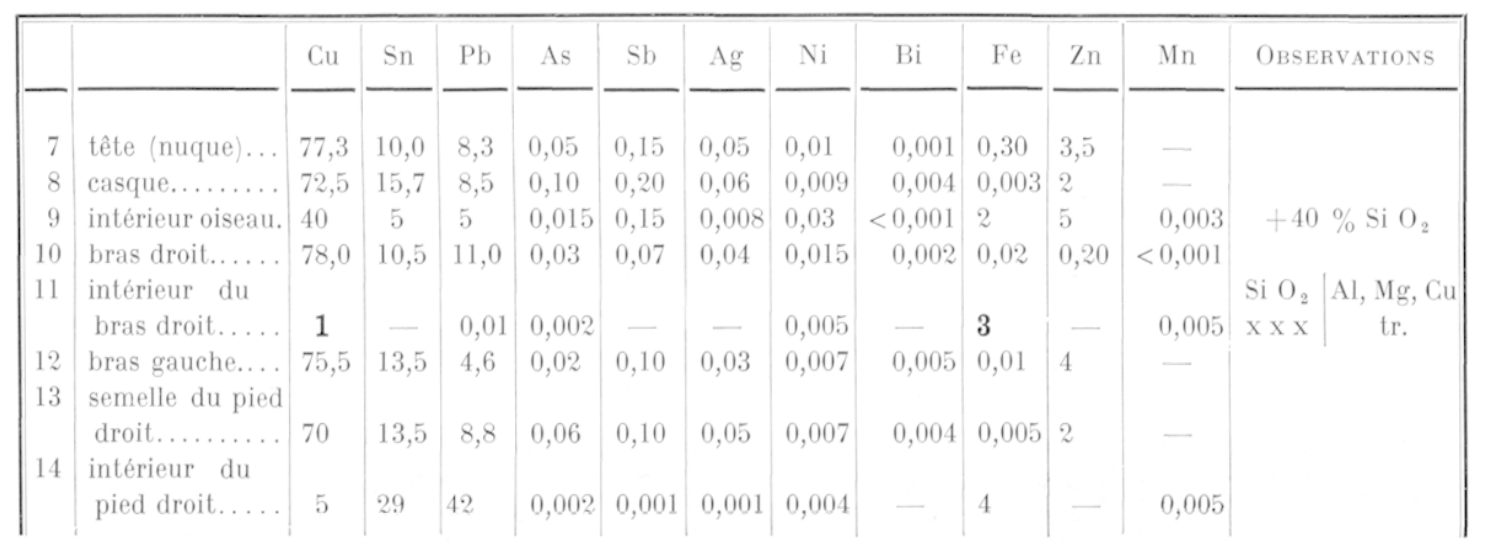

On peut se demander, devant ce visage, si ce n'est pas le portrait, au réalisme à peine idéalisé, d'une jeune fille de l'Armorique antique, encore que rien ne prouve l'origine locale de la statue. Sur un cou gracieusement évasé et naturel, la tête fine est celle d'une adolescente de quinze à seize ans (fig. 3). On ne peut quère douter qu'il s'agisse d'une jeune fille, malgré certains avis contraires qui y voient un jeune guerrier, ou même le "Chevalier au Cygne». La forme du visage est un ovale régulier qu'accentuent des joues pleines et lisses, aux pommeltes peu marquées ${ }^{18}$. Le menton, rond et charnu, laisse deviner la naissance d'un léger double-menton, trait que l'on retrouve dans d'autres masques en bronze de la Gaule (fig. 9). La bouche, bien modelée, tombe légerement aux commissures, ce qui contribue à donner à la physionomie une expression de mélancolie. La grouttière labiale est correctement marquée. Le nez, qui porte la trace du rhoc provoqué par le soc de la charrue au

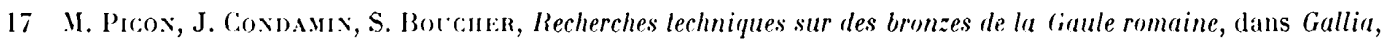
XXIV, 1966, p. 212-215; XXI; 1967, p. 159-160; XXVI, 1968, p. 269-272.

18 Haut. totale, du sommet du cimier a la base du cou : $18,2 \mathrm{~cm}$; haut. du masque avec le cou, : $11,5 \mathrm{~cm}$; haut. de la tête : $9,2 \mathrm{~cm}$; haut. du visage : $7,3 \mathrm{~cm}$; de la base du nez au menton : $3,7 \mathrm{~cm}$; de la base dı nez aux au bas de la chevelure $: 3,7 \mathrm{~cm}$. 


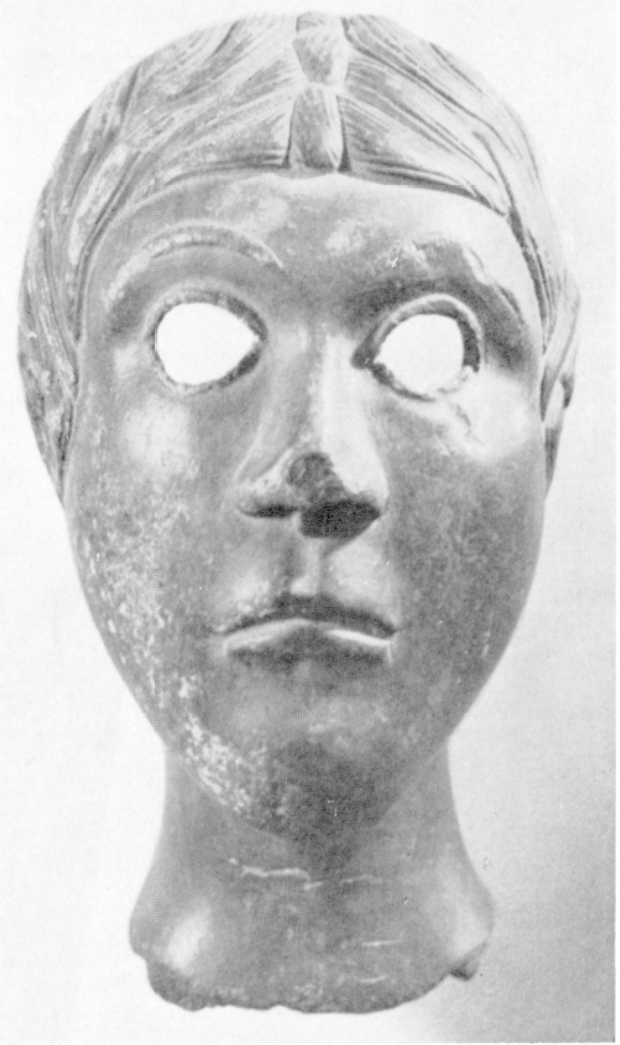

4 a

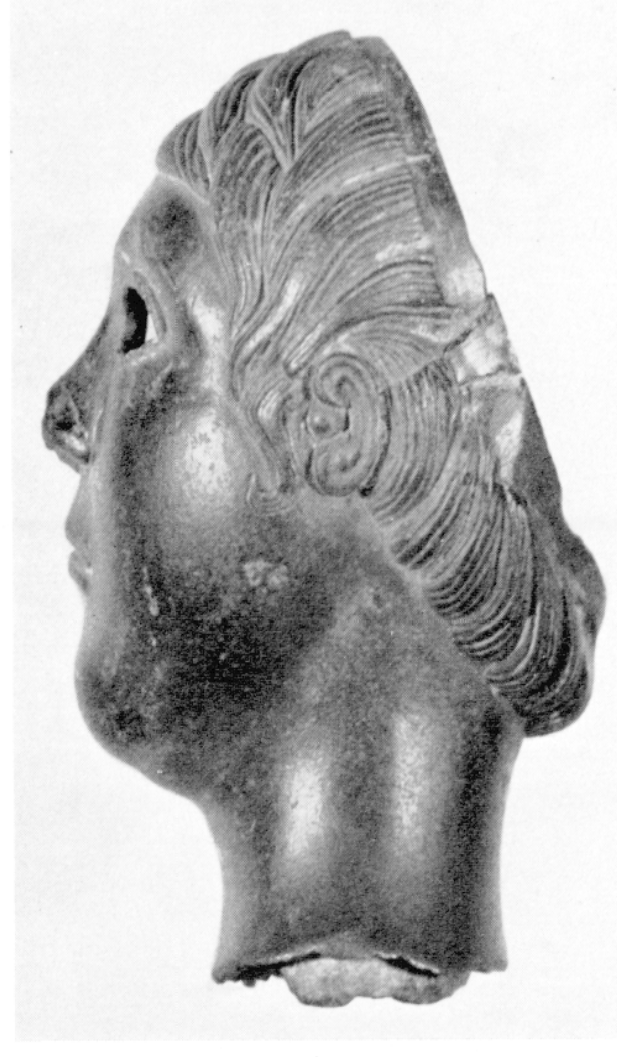

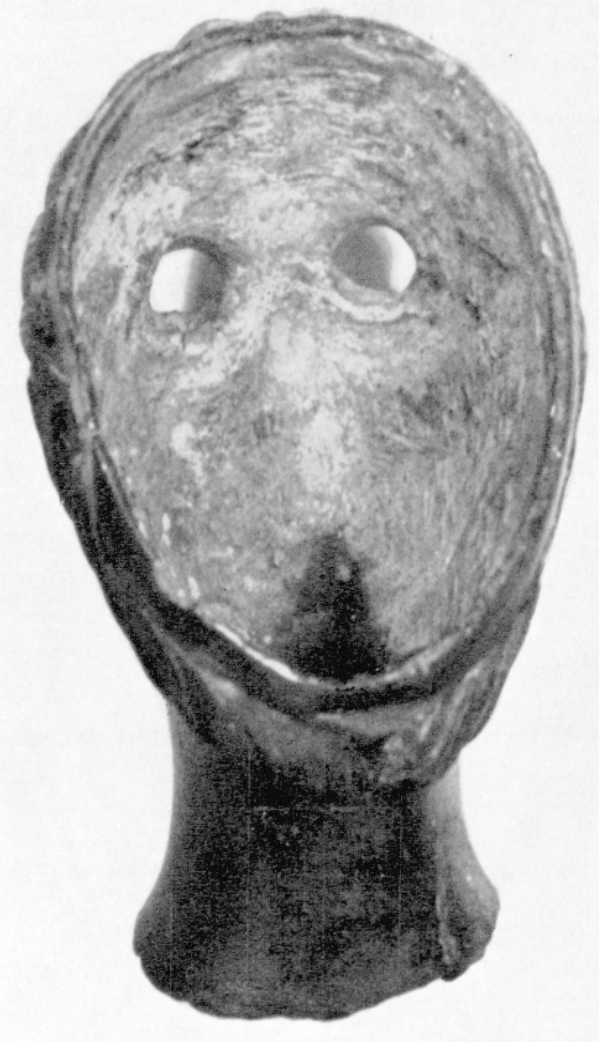

4 b

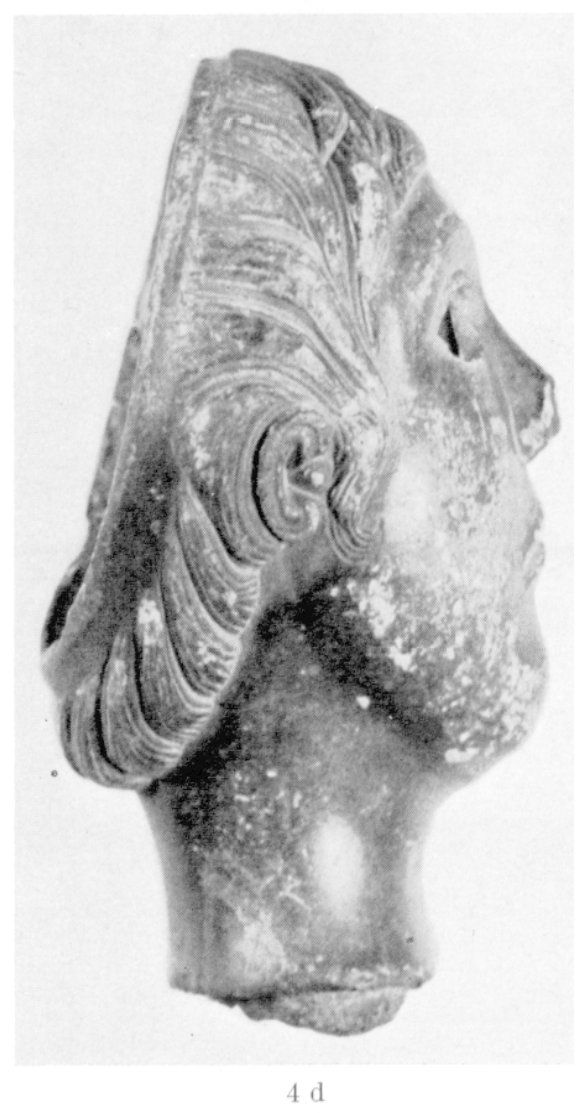

$4 \mathrm{~d}$

4 La tête. 
moment de la découverte, est régulier, triangulaire, en forme de pyramide à trois faces. A la base, les deux narines sont figurées en léger creux et le mouvement des ailes du nez est perceptible. Les bourrelets sourciliers sont indiqués par un léger relief, sans traits de poinçon pour représenter les sourcils. Comme il arrive souvent, les yeux manquent: on peut penser qu'ils sont restés au fond de la cachette ou qu'ils ont été projetés au loin lors de la découverte. Faits, selon toute vraisemblance, en pâte de verre, ils étaient habituellement fixés à l'intérieur du masque par du plomb fondu (ici, cependant, nous n'avons trouvé aucune trace de soudure). De fines paupières délimitent des orbites agrandies. Le front est bas et quelque peu fuyant. L'architecture générale du visage, vu de face, est harmonicuse, l'expression de la bouche, le regard lointain, composent une physionomie hiératique, plus propre à la représentation d'une divinité que d'un personnage vivant. On peut remarquer que la partie inférieure du visage occupe une place importante, a tel point que le nez se trouve exactement au milieu de la face, a égale distane de la pointe du menton et du départ des cheveux.

Le profil est moins heureux : la tête apparait trop etroite, le menton prend trop d'importance (fig. 3 et 4 ). La ligne n'obéit pas aux canons classiques : le front bas, le nez retroussé, le menton lourd sont loin du profil grec et évoquent plutôt l'esthétique du visage au Bas-Empire. L'oreille est curieuse : alors que, par ailleurs, nous avons pu noter l'exactitude anatomique de certains éléments, comme la bouche, le nez, le menton, par contre, l'oreille est un simple signe, réduit à une double ligne spiralée enserrant une perle, dessinant une sorte de pella. Le lobe inférieur est de même dimension que le lobe supérieur, ce qui contrarie la réalité. Petite, placée haut, presque au niveau des yeux, elle fait corps avec la tête et le pavillon, n'est pas décollé, comme sur la plupart des portraits ${ }^{19}$.

De toutes les parties de la tête, la coiffure est certainement celle que l'artiste a réalisée avec le plus de soins (fig. 4). Chaque mèche, bien isolée des autres, est formée d'un relief moulé sur lequel on a ajouté, au burin, dans un style linéaire, l'indication des cheveux. L'ensemble de la coiffure forme une calotte enserrant le crâne, tombant très bas sur le front. L'absence de la partie centrale, du vertex notamment, nous empêche de bien comprendre son agencement. De part et d'autre d'une raie médiane, cachée par trois mèches superposées ou par une tresse, s'ordonnent des mèches souples qui disparaissent sous d'autres plus longues, ramenées en avant et couvrant les pariétaux. Sur la nuque, les mèches sont relevées en un bourrelet léger. Les cheveux formaient-ils un chignon, caché par le casque? il ne semble pas. Nous constatons simplement que, sur le front, on voit uniquement des pointes de mèches et, sur la nuque, des racines de cheveux. La solution est peut-être donnée par la mèche qui enserre l'oreille : elle prend le départ derrière l'oreille, en fait le tour, passant sous une mèche venue de derrière et se termine par devant, en une courbe contrariée, dont l'extrémité est gravée au burin. Chaque mèche a pu être traitée de cette manière et tous les cheveux ramenés d'arrière en avant, depuis la nuque jusqu'au front. La présence de mèches sur le devant de la tète

19 L'oreille est décollée chez le jeune Aquitain, cf. Espéraxdnec, outr. cilé, no 7055; le jeune homme de Prilly, cf. Espéraxdieu, ouvr. cité, no 5383 , etc. L'oreille massive se trouve rarement sur des aruvres de qualité et toujours en marbre ou en pierre. 
RENÉ SANQUER
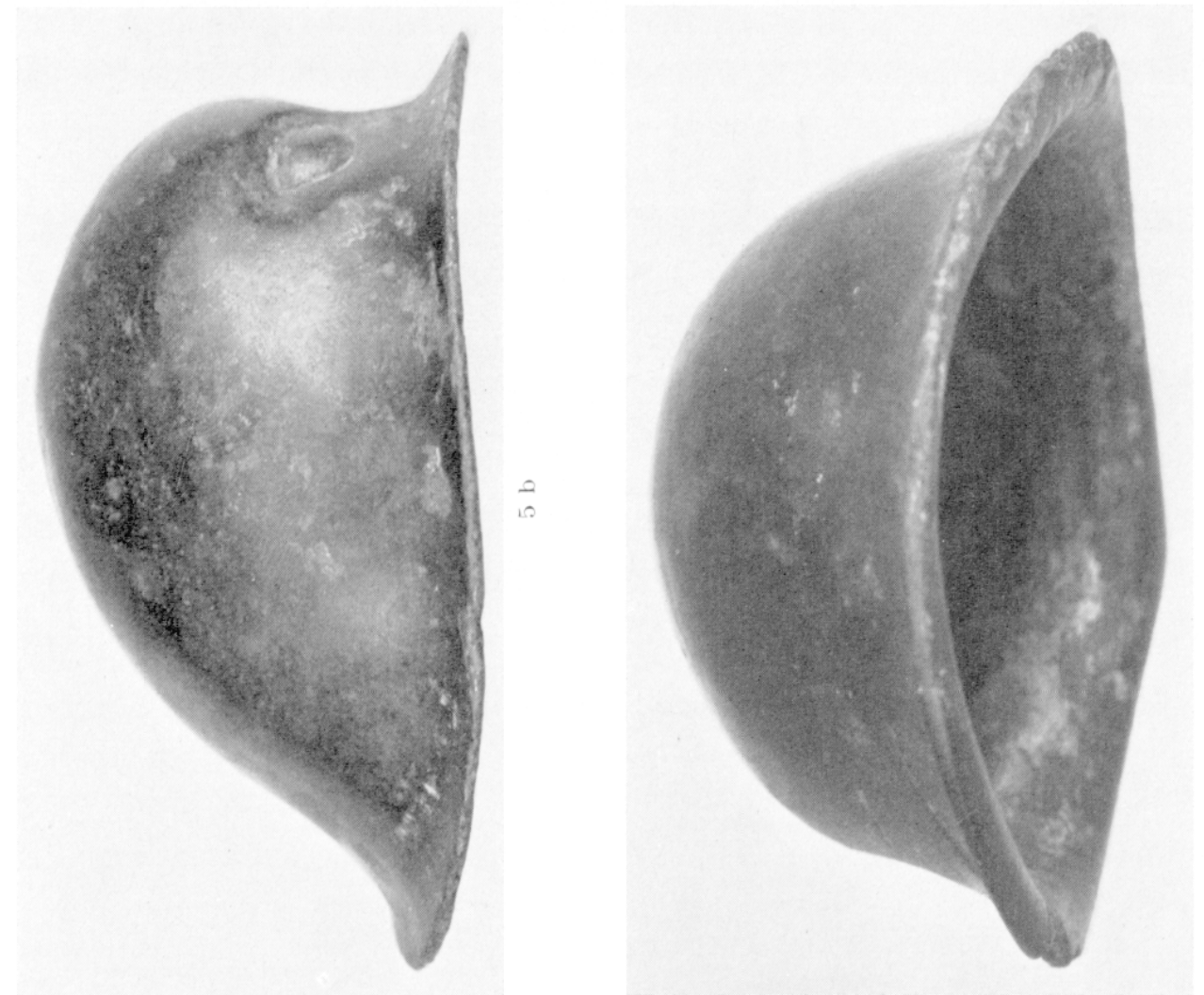

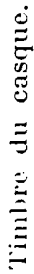
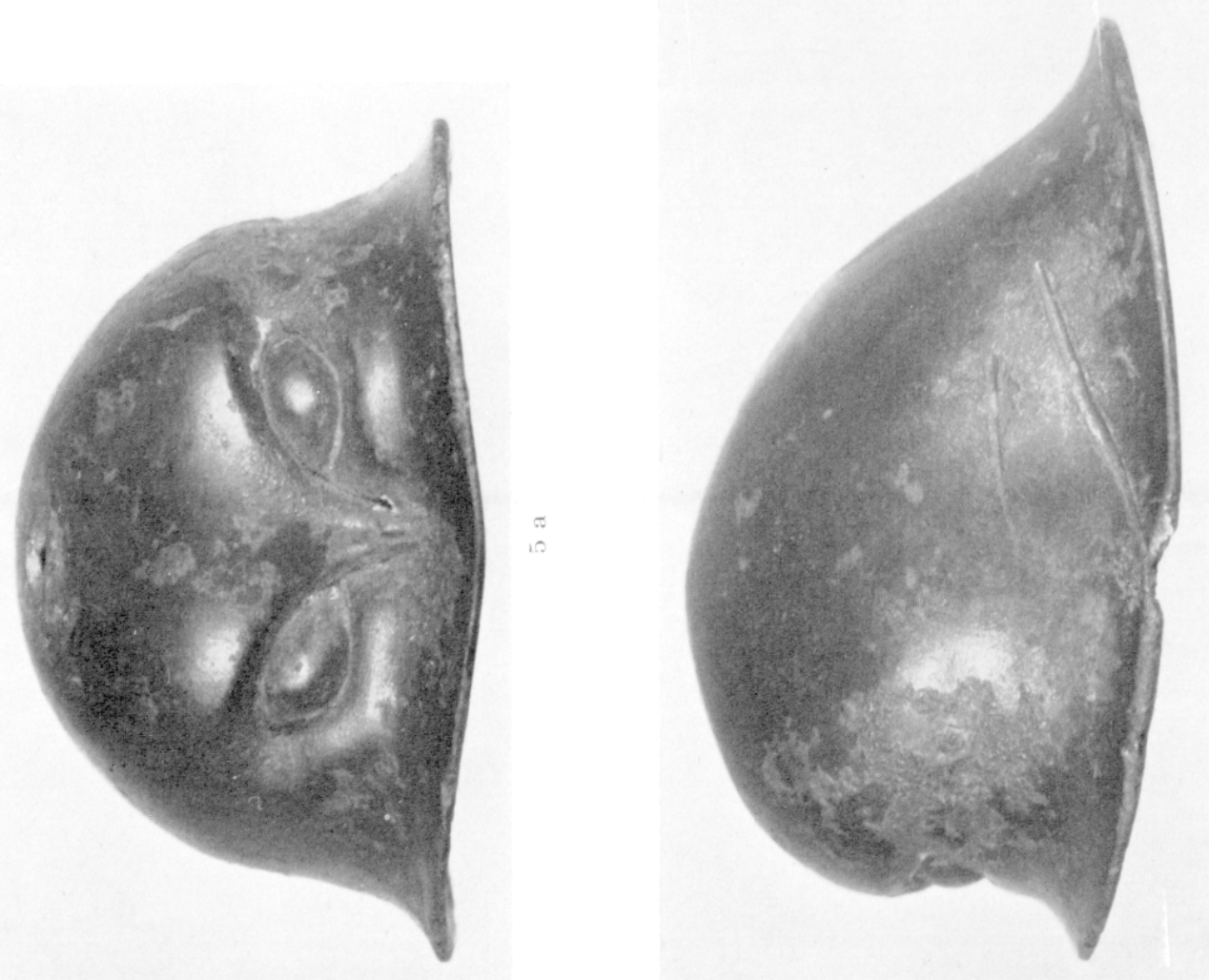
a pu faire penser qu'il s'agissait d'une roiffure masculine. Nous ne doutons pas que ce soit une coiffure de femme, mais nos recherehes ne nous ont pas permis de trouver, dans toute la sculpture antique, un agencement identique.

Le casque complet se compose de la bombe et de ses appendires, le cimier et le portecimier (fig. 3202. A l'origine, sclon toute varisemblance, une crite de plumes, fixée sur le cimier, complétait l'ensemble. La bombe, du type dit en cloche, avec un timbre hémisphérique aux rebords évasés, conserve encore, sur le devant, le tracé du visage du casque corinthien, evoquant en mème temps la chouette d'Athéna (fig. j). Il ne peut se rabaltre sur la figure et ressemble, à s'y méprendre, au casque de quelque armée moderne. Lin oiseau fait office de porte-cimie ${ }^{21}$. Arec un grand souri d'exactitude naturaliste, l'artiste l'a saisi au moment de l'envol, à l'instant où il décolle, le con tendu mais encore souple, les ailes redressees et serrées, non encore déployées (fig. 6). Le dessin des plumes respecte également la réalité, avec quelque raideur cependant : les rémiges at les plumes de couverture, hachurées au burin, sont disposés sans souri de parallélisme absolu. L'identité de cet oiseau n'est pas tout à fait assurée. Notre choix oscille, parmi les palmipèdes aquatiques, entre l'oie saunage et le cygne. Les ornithologues consultés ont opté pour le cygne sauvage, car le cou de l'oie saurage est plus court, colui du cygne blanc, plus long. Quel que soit cet oiscan, la place importante qui lui est réservée dans le couvre-chef n'est certainement pas fortuite. Ie cimier, du type bifide et long, forme en section une gorge en $U^{j}$ assez profonde, dans laquelle a pu trouver place, jadis, une aigrette de plumes véritables, fixée à la cire. Sa courbe harmonieuse, terminée par trois petites boules (l'une manque), est soulignée par des traits courts qui éroquent le départ. de l'empennage (fig. 3).

Les membres, quoique d'exécution habile, montrent une disproportion marquée par rapport à la tète et les uns par rapport aux autres (fig. 1) : les membres supérieurs, trop petits, ainsi que les pieds, et le bras droit plus fort que le bras gauche, laissent planer un doute sur l'équilibre de l'œurre complète, à tel point que certains ont pu se demander s'il n'y avait pas là, réunis par le hasard, les éléments de plusieurs statues. Les différences dans la composition du métal, ainsi que certains détails anormaux du costume ${ }^{22}$, ne viennent-ils pas à l'appui de cette supposition? Lan fait, la réunion d'un bras droit et d'un bras gauche, d'un pied droit et d'un gauche, ne peut être fortuite, et les circonstances mêmes de la découverte prouvent qu'il n'y avait qu'une seule statue. D'ailleurs, on sait que des cuvres de second ordre offrent de nombreux exemples de déformations et d'erreurs de proportions.

Le bras droit est fléchi à angle droit, la main refermée sur un objet cylindrique, pour soutenir une haste ${ }^{23}$ (fig. 7 a). Le bras gauche est a demi tendu vers le bas et la main entrouverte devait tenir un objet léger, une patère plutòt qu'un bouclier (figg. 7 b). Il n'en reste cependant aucune trace. Dans les deux cas, malgré la disproportion déjà notée, la facture est la mème : les bras charnus laissent peu deviner les muscles, mais l'articulation

20 Haut. totale : $22,6 \mathrm{~cm}$; long. du casque seul : $10,2 \mathrm{~cm}$.

21 Jong. : $8,7 \mathrm{~cm}$.

22 Ainsi les pieds totalement decouverts.

23 Long. du bras droit : $12,4 \mathrm{~cm}$; long. du bras gauche : $15,8 \mathrm{~cm}$. 


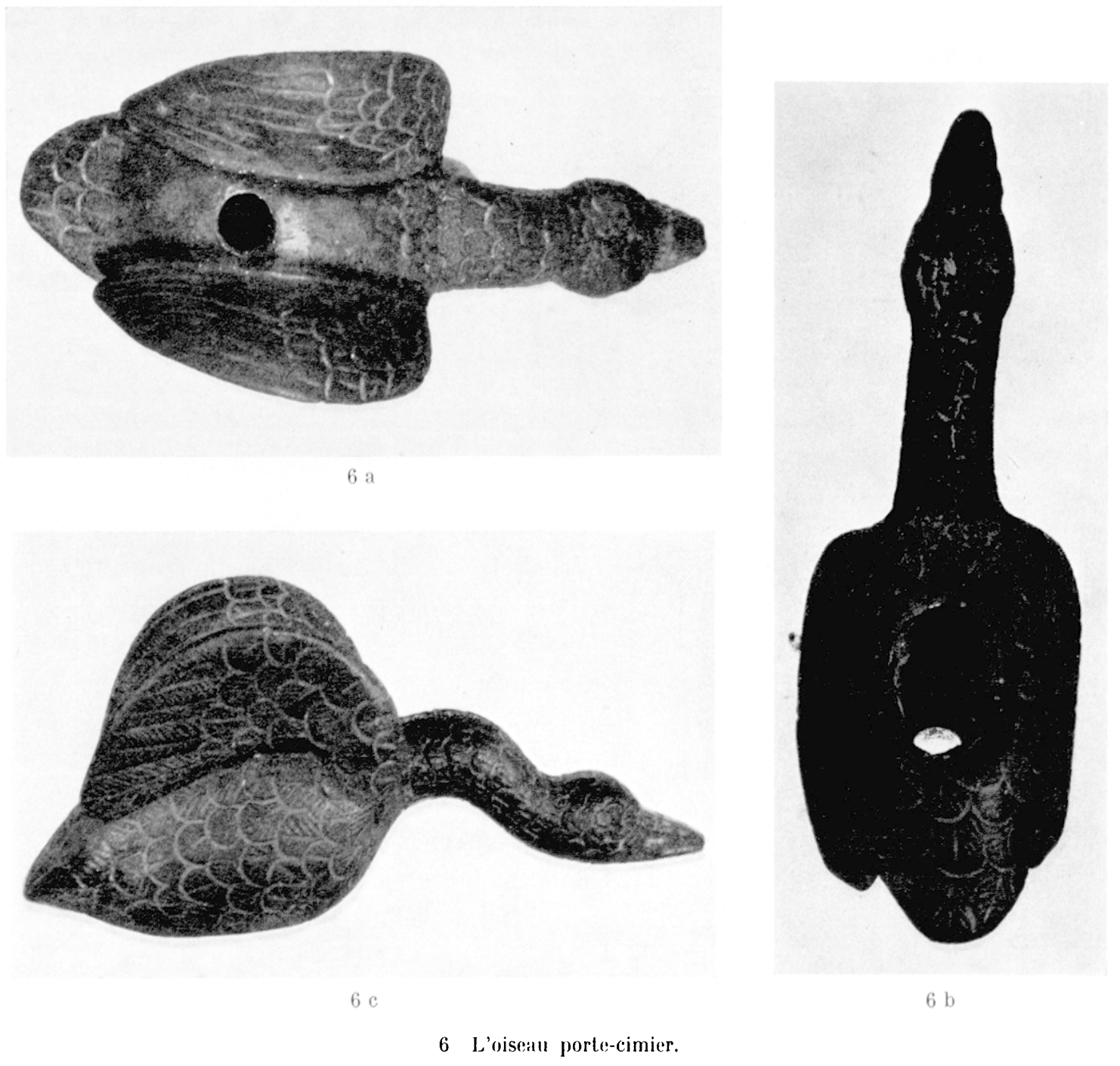

du courle fait une légère saillie. Les doigts fins et longs, serrés les uns contre les autres, sont exactement reproduits, ongles compris.

Les pieds portent une chaussure à semelle épaisse et plate ${ }^{24}$. L'empeigne recouvre entièrement le pied; un contrefort, fermé par une agrafe (l'agrafe de la chaussure droite manque), enveloppe la cheville et le talon. Le pied gauche, posé à plat, supportait le poids du corps (fig. 8 a). Le bas de la jambe apparaît au-dessus de la chaussure, révélant que la robe n'atteignait pas tout à fait la cheville. Le pied droit, en demi-flexion, met en évidence un léger déhanchement du corps (fig. 8 b). La statue était posée sur un socle

24. I.ong. du pied droit : $7,5 \mathrm{~cm}$; long. du pied gauche $: 7,3 \mathrm{~cm}$; haut. de la chaussure droite : $2,8 \mathrm{~cm}$; haut. de la chaussure gauche : $2,5 \mathrm{~cm}$. 


$$
\begin{aligned}
& 78 \\
& 35
\end{aligned}
$$




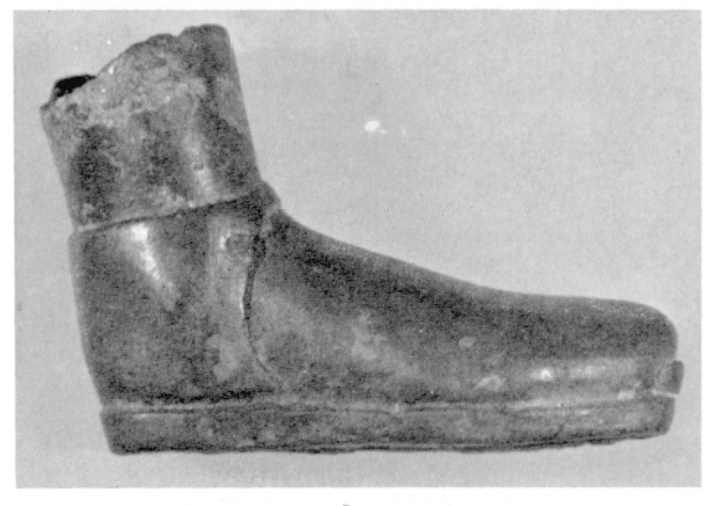

8 a

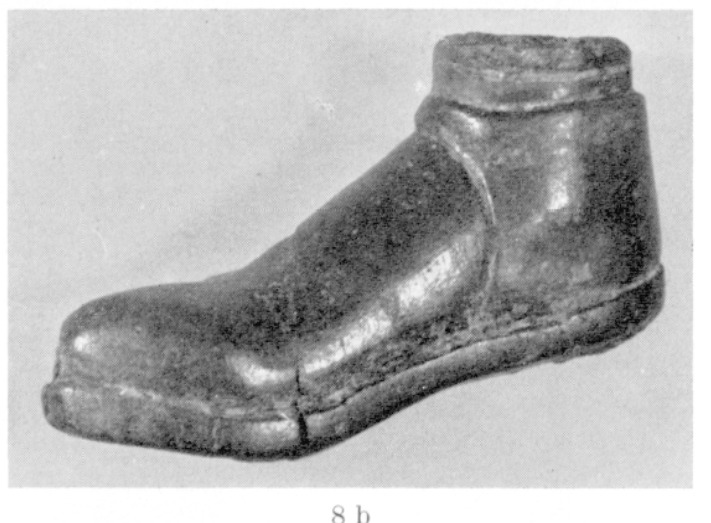

b, pied droil.

car la chaussure gauche, à plat, porte une semelle évidée, tandis que la droite, à demi soulevée, offre à la vue une semelle pleine à l'arrière, alourdie sur l'avant par un alliage au plomb, qui a fait éclater le bronze.

Telle est cette ouvre, rare par sa qualité et le fini do tous ses éléments, insolite par le lieu de sa trouvaille ${ }^{25}$. Elle appartient pourtant à un groupe de statues et de statuettes en bronze fondu, dont l'ensemble n'a pas encore été étudié. On pourrait en citer en Angleterre ${ }^{26}$, mais c'est sur le territoire de la Gaule que la plupart de ces objets ont été découverts.

Nous avons montré plus haut que, même avec des œuvres d'inspiration tout à fait classique, mais exclusivement trouvées en Gaule, un parallèle technique était possible sur un point au moins, l'absence de calotte crânienne. Il en est de mème sur le plan du style : malgré des techniques tout à fait différentes, des dates fort variées, des matériaux divers, on peut isoler quelques traits communs dans des œuvres comme le portrait masculin de Bordeaux, la "tête Danicourt ", trouvée près de I.yon, le buste d'Évreux, les masques

25 F. Bramemer art. cilé, 1969, 1, p. 101, publie une carte de répartition des points de découvertes des sculptures en metal battu et repousse exécutees par des orfevres et des chaudronniers. On peut voir que l'Armorique en est absente. Statues et masques sont surtout groupés au centre de la Lyonnaise et le site le plus proche est celui de Notre-Darne-d'Allençon (Maine-et-Loire).

26 Masque de Charterhouse-on-Mendip, Somerset, Angleterre Muse de Bristol, cf. E. T. LExds, Cellic Ornament in the British Isles down to A.J). 700, 1933, p. 97, figr. 29 b et S. Prociotr, Early Cellic Art, Edimbourg, 1970, p. 16 et $\mathrm{n}^{\circ} 80$. Iaut. : $86 \mathrm{~mm}$. Masque tronvé "quelque part en Angleterre "et actuellement au Musée national de Copenhague. Haut. : 12,8 cm. Cf. O. KLindt-Jexsex, Acla arch., XX, 1949, p. 118-119, no 315; \$. Piggott, Antiq. Journal, 1963, p. 116-118; J. .I. C. Toyxise, Art in Britain under the Romans, 1964, p. 110, note 2, IX, pl. XXVIII b ; J. V.S. IEsiaw, Art of the European Iron Age, 1970, p. 144 et n ${ }^{\circ} 240$. La tête masculine de pierre de Gloucester, du Musée de Gloucester, cf. J. F. Rmodes, Calalogue of Romano-Brilish sculplures in the Gloucester Cily Museum, 1964, n 3, publiée par J. V. S. Megaw sous le no 2.3, appartient au même style. Le traitement des meches de cheveux et le dessin de l'oreille offrent quelque parenté avec la statue de Dinéault. Elle est datée du milieu du I ${ }^{\mathrm{er}} \mathrm{s}$. 
de Garancières-en-Beauce, de Montserrié, de Notre-I)ame-d'Allençonn. Lne même simplification des lignes et des plans, un certain hiératisme, une expression sévère, les caractérisent toutes. Cependant, les œuvres qui allient la parenté technique et la parenté stylistique avec la statue de Dinéault sont peu nombreuses. Le dieu de Bouray, déjà cité, en est assez voisin mais les rapprochements les plus étroits peuvent être tentés avec la série de têtes de bronze trouvées au siècle dernier en forêt de Compiègne.28. La technique de fabrication présente un certain nombre de similitudes. Jeux têtes de jeunes filles surtout se détachent du lot et peuvent être comparées à celle de Kerguilly en l)inéault ${ }^{29}$ : de dimensions voisines. réalisées en fonte creuse, elles devaient ètre, à l'origine, placées sur un corps (figg. 9) a. b), Les globes oculaires sont en pâte de verre et fixés au plomb; la calotte crânienne, ouverte, est obturée par un couvercle mobile. L'architecture du visage offre également des points de comparaison : les têtes, vues de profil, ont la mème étroitesse, le menton lourd; le nez est placé au centre exact de la face et la partie inférieure occupe près de la moitić du visage. Cependant, comme dans le cas du dieu de Bouray, le traitement est beaucoup plus sommaire : le cou est lourd, cylindrique, presque aussi large que le crâne, les principaux traits du visage sont réduits à l'essenticl, la chevelure, stylisce à l'extrème, l'expression, irréelle. La statuette de Kirguilly montre un art beaucoup plus élaboré et réalise une synthèse heureuse antre un art indigène encore fruste et un apport méditerranéen plus évolue sur le plan de la technique et de la forme. Nous manquons de criteres archéologiques pour dater ces curres. Les historiens s'accordent cependant à ne pas faire remonter l'apparition de ce type d'ouvrages en-deçà de la période romaine, et la plupart préfèrent placer la majorité des œuvres au $\mathrm{I}^{\mathrm{er}}$ siècle apres J.-C., quand ce n'est pas au $\mathrm{II}^{\mathrm{e}}$. Les masques de Notre-1)ame-d'Allençon, qui appartiennent nettement à cette école, ont été datés du ${ }_{\text {III }}{ }^{\mathrm{e}}$ siècle après J.-(., ce qui repousse presque jusqu'au Bas-Empire l'influence de ce style.

Pouvons-nous tenter, malgré tout, de situer la statue de Dinéault, avec plus de précision à l'intérieur de la période romaine? Le lieu de la trouvaille ne peut être d'aucun secours : aucun vestige des premiers siècles de notre ère n'a été recueilli sur le territoire de la commune, qui semble aroir été fréquenté plutôt à l'époque gauloise. Ln sondage à l'emplacement présumé de la cachette n'a donné aucun résultat. Quant à l'objet lui-mème, il offre, par certains de ses aspects, une possibilité d'appréciation. Le casque et ses accessoires constituent un élément de datation, dans la mesure où l'archéologie et la sculpture nous ont laissé des témoignages assez nombreux pour nous permettre de suive l'évolution de cette pièce d'armure. Le type de casque représenté ici est appelé, par certains, étrusco-

27 Pour le portrait masculin de Bordeaux, voir R. I.axter, Tèle d'un jeune chef aquitain, dans .Monuments...

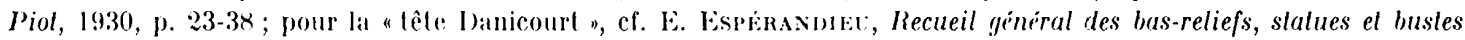

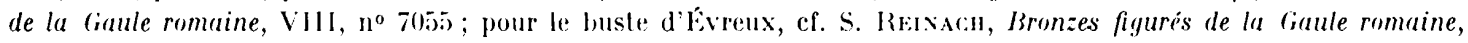
Paris, 1894, p. 2.10; pour le masque de Garancieres-n-Beauce, voir Ir. I. axtrix, line tile de bronze antique provenant de Garancieres-en-Beauce, Eure-et-Loir, conservée au Musée de Chartres, dans Bull. de la Soc. nat. des Anliquaires de France, 1934, p. I 66 . Pour le masque de Jontserie, voir F. Bnakmer, arl cite, 1969, 1, p. 82-83; pour les masques

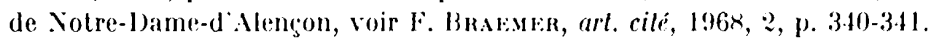

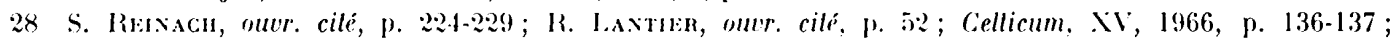
J. V. S. MEGaw, oluvr. cité, no 239, p. 144.

29 Jos d'inventaire an Musie des antiquites nationales de Sant-Germain-en-Laye : 27.460 et 27461 . Cf.

- Resach, ouvr. cité, nos 217 et 220 . Jimensions : 0,10 el $0,097 \mathrm{~m}$. 
corinthien, par d'autres, romano-corinthien, et marque l'aboutissement de l'évolution du casque de l'époque grecque classique. Encore utilisé par certains légionnaires à la fin de la République, il apparaît pour la première fois sur l'autel dit de Iomitius Ahénobarbus et on le voit sur d'autres bas-reliefs ${ }^{30}$. Mais, d'une part, la date de ces sculptures est encore discutée ${ }^{31}$ et, d'autre part, dans le cas d'une divinité, un type de casque archaïque a pu se perpétuer au-delà de son utilisation par les troupes. Le casque, donc, ne nous aide guère. Du vêtement, il ne reste que les chaussures. Le modèle de celles-ci se rapproche de celui qui chausse souvent le dieu Sucellus-I)ispaler ${ }^{32}$, mais nous n'avons pas retrouvé le type exact de la sandale représentée ici.

L'élément le plus caractéristique, en définitive, est encore la coiffure. Il est habituel de considérer que la coiffure en forme de calotte enserrant étroitement le crâne, répartie en mèches souples, dont le détail est fermement dessiné, dans un style purement linéaire, dérive d'une mode romaine de la fin de la République popularisée notamment par les portraits d'Auguste et de ses contemporains. Elle se maintient, en se simplifiant, au moins jusqu'au règne de Claude et recourre en fait la première moitié du I $^{\text {er }}$ siècle. Cépendant, nous n'avons trouvé aucune coiffure exactement semblable à celle de la petite déesse de Dinéault. Aucune impératrice romaine, en particulier, ne l'a portée. Est-ce une coiffure provinciale, propre a la Gaule? N'est-ce pas, plutôt, une coiffure d'enfant? Dans ce cas, on pourrait voir, dans les mèches qui recouvrent la raie médiane, le rappel de la tresse qui ramenait d'arrière en avant les cheveux longs des enfants, au ier siècle ${ }^{33}$. Mais comme les coiffures enfantines se sont perpétuées, une certaine prudence s'impose. Cependant, comme il faut proposer une date, nous suivrons l'opinion courante qui fixe à la première moitié du rer siècle l'apparition et le développement de cette catígorie d'ouvres, qui correspond, en gros, à celle que propose F. Braemer ${ }^{34}$ pour le dieu de Bouray.

Dans la plupart des cas, l'absence d'attributs classiques, trouvés en relation aver les masques ou les bustes, empêche toute identification de la divinité représentée. Tel n'est pas notre problème. Nous pouvons, sans mal, reconnaître la divinité méditerranéenne dont on a pris l'habit. C'est, évidemment, Athéna ou Minerve, mais cela ne résout pas l'énigme de la divinité locale sous-jacente, celle que l'inlerprelalio Romana assimilait à Athéna-Minerve en Gaule. Il est difficile de croire, en effet, si l'on admet une datation haute, la plus probable, que l'on vénérait dans l'A rmorique occidentale pendant les premiers

30 P. Cotissix, Les armes romaines, Paris, 1926. J. Haruaxo, L'armée el le soldat à Rome de 107 à j0 avanl notre ère, laris, 1967, p. 71, fig. 1. Co modele figure egalement sur lautel du l.ouvere sur la ciste augusteenne de: Préneste.

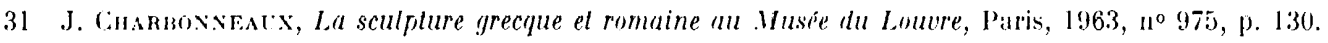

32 Voir la staturlta de Dispaler-Sucellus du Musé Calvet d'Avignon, H. Rondaxn, Bronzes anliques de HauleProvence, XVIII suppl. à Gallia, 1965, p. 32, no 12.

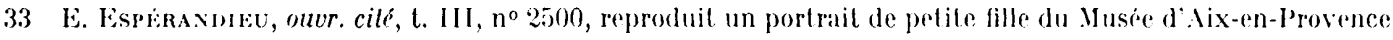
portant une tresse de ce type. Il faut noter cependant que le bourrelet forme par la tresse est plus large et plus epais que dans la coiffure de Kerruilly.

34 F. Bnakmir, art cilé, p. 86-88, date le dieu de Bouray, d'apries la coiffure, du regne de Claude. I.a definition que donne J.-J. HATr, Sculplures gauloises, Paris, 1966, p. 40, s'applique trés exactement à la statue de Kerguilly : "l'art du bronzier indigene produit encore, au cours du regne d'Auguste, des chefs-d'ouvre remarquables dont les caracteres complexes montrent la rapidite d evolution el la faculte dassimilation des maitres gaulois : linfluence du portrait romain est déjà sensible, les traits du visage sont rendus avec naturel... : l'artiste indigene s'est humanisé et a su se meltre au diapason des techniques qui lui etaient apporties d'latien. 
siècles de notre ère une divinité gréero-latine. (On connaît mal l'arrière-plan archéologique de cette époque, mais il est certain que les influences méditerranéennes n'y avaient encore pénétré que très faiblement. Les monnaies de billon, imitées de celles de l'Indépendance, ont circulé au moins jusqu’à la fin du règne d'Auguste et les quelques habitations connues, ainsi que la céramique commune, montrent la survivance des techniques de La Tène III. Seuls quelques tessons d'amphores méditerranéennes et de très rares poteries d'Arezzo témoignent ici d'une pénétration des influences romaines. Il faut attendre le règne de Claude, aver la construction de routes, pour noter un début d'assimilation, qui n'aura véritablement d'effet qu'au $\mathrm{II}^{\mathrm{e}}$ siècle après J.-(:. Nans ces conditions, on ne voit guère comment une Athéna-Winerve purement méditerranéenne aurait pu ètre adoptée en Armorique. Bien qu'il nous reste fort peu du vètement de la statue, on peut remarquer cependant que la robe de la déesse ne recouvrail pas les chaussures et s'arrêtait au-dessus des chevilles. C'est là une anomalie car, dans tous les cals, le rêtement féminin antique atteint le sol, recouvrant à demi les chaussures. Seule, parmi les divinités, Artémis-IDiane porte une tunique courte. Enfin. le choix du cygne, pour orner le casque de la déesse, n'est certainement pas fortuit, ru l'importance qui lui est donnée, et la qualité de sal facture. Or, il n'est jamais l'attribut d'Athéna dans ses representations dassiques ${ }^{35}$. Il se peut done que la Minerve de l)inéault ait été légèrement différente de son modèle grécoromain.

Il n'est pas interdit de proposer quelques rapprochements. Cisar nous renseigne sur les dieux de la Gaule ${ }^{36}$. A côté des quatre grands dieux masculins, qu'il désigne par leur homologue romain : Mercure, Apollon, Mars et Jupiter, une seule divinité féminine est citée, Minerve, qui "enseigne les rudiments des arts et des métiers". Il est admis qu'il ne s'agissait pas de la Minerve vénérée, à cette époque, à Rome et en Italie, mais d'une grande déesse indigène, aux fonctions à peu près équivalentes. Les historiens de la religion celtique ont bien souvent établi un parallèle entre Athéna-Mlinerve et la Brigil ou Birgil des textes irlandais du Haut-Iloyen Age èz déesse universelle des Celtes. Ln témoignage épigraphique et artistique, dans un pays voisin, à substrat celtique, illustre la complexité de ces questions : une stèle découverte à Birrens, près du mur d'Hadrien, en 1731, et conservée aujourd'hui au Musée national d'Érosse a Edimbourg, porte une dédicace a une déesse Brigantia, divinité tutélaire des Brigantes, peuple établi au sud de la chaìne pennine, en Bretagne ${ }^{38}$. Le bas-relief qui surmonte l'inscription représente une divinité casquée, vêtue d'un long chilon et d'un manteau, portant l'égide. Elle tient dans la main droite une lance, un bouclier est posé près de sa jambe gauche (fig. 10) : il est clair que ce sont la les attributs de . Vinerve. Pourtant, le casque à panarhe, orne de cornes, est bordé

35 Le cimier du casque d'Athena-.Vinerve est tris souvent supporte par un oistau, cf. S. Rivacin, Répertoire

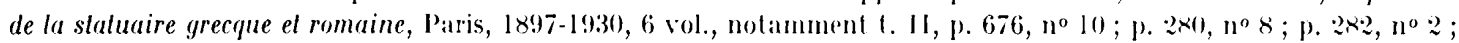

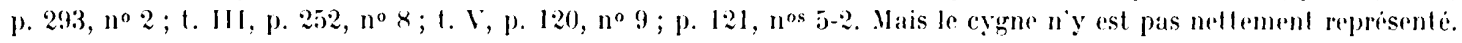
36 De bello gallico, $\mathrm{VI}, \mathbf{5 7}$.

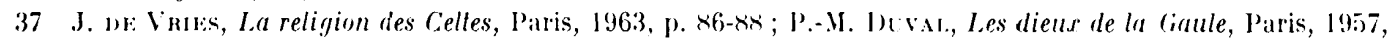
p. 9.4 avec un point d'interrogation cependant .

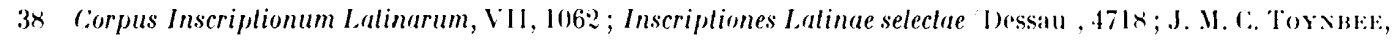

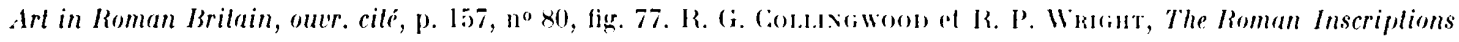
of Brilain I, inscriptions on stone, (0xford, 1965, 1. 640, n० 20191, pl. XIX. 


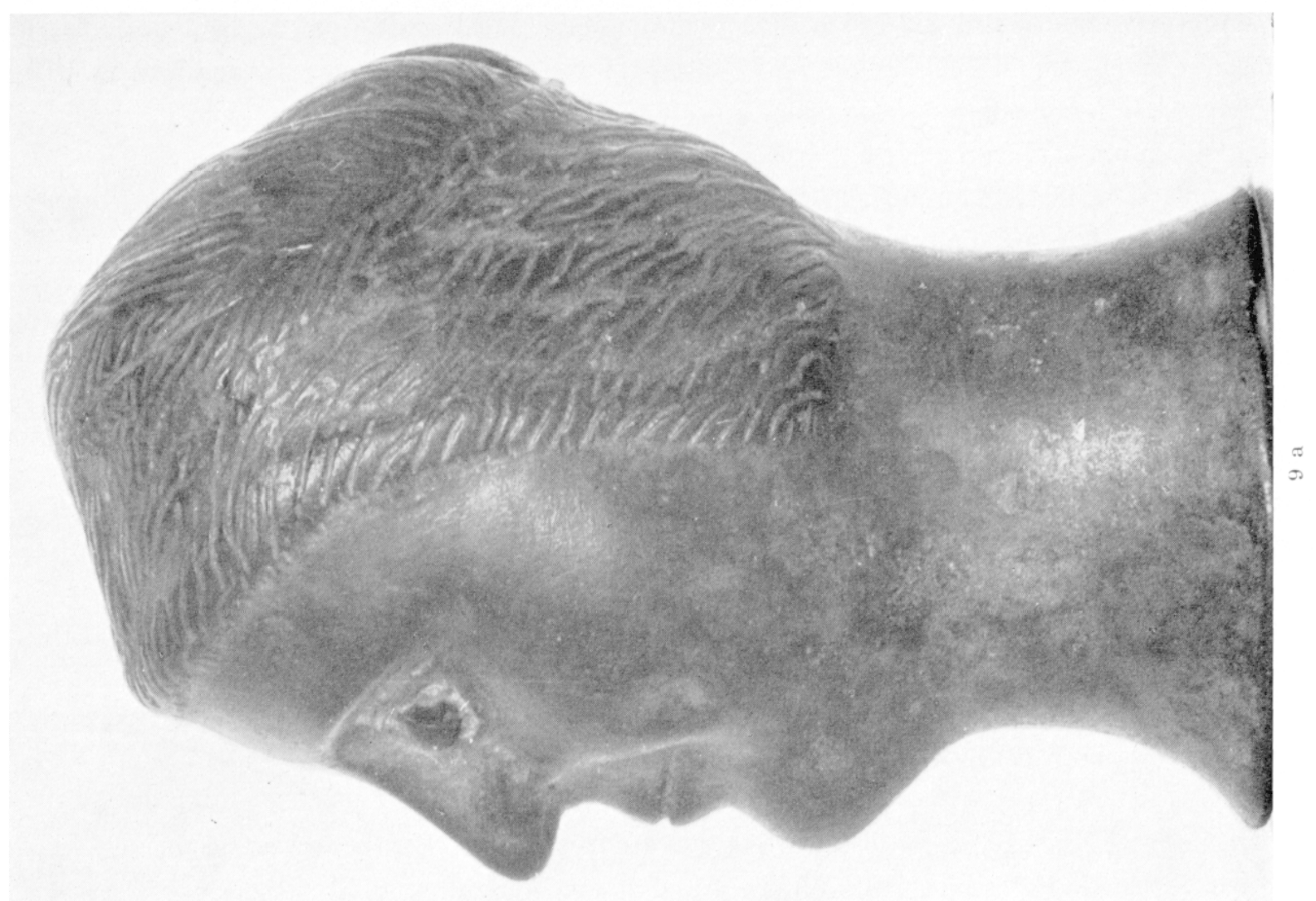

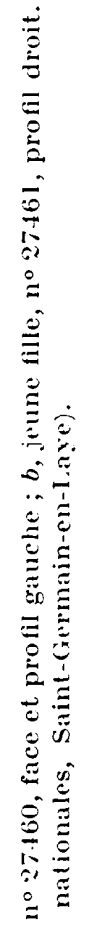

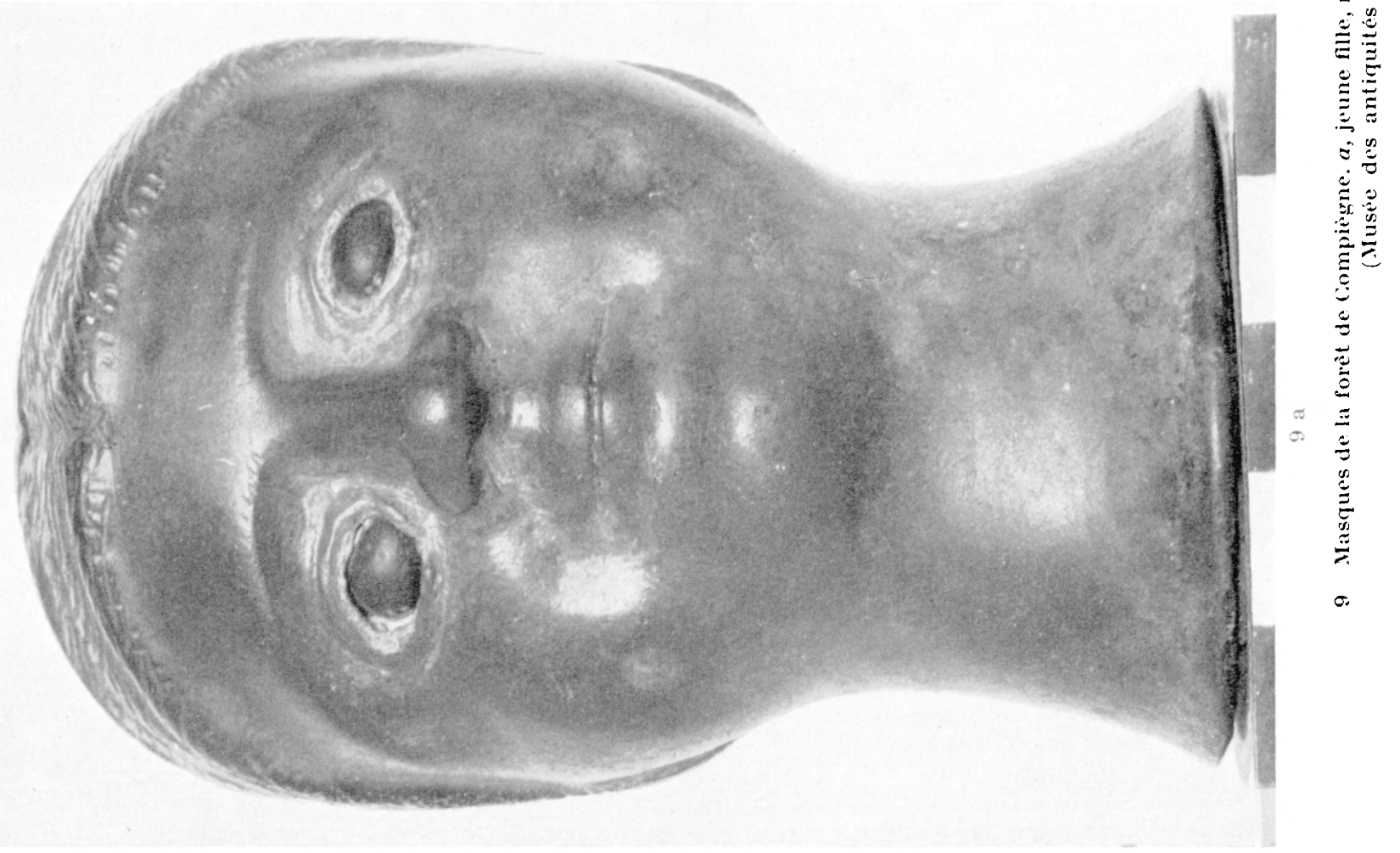



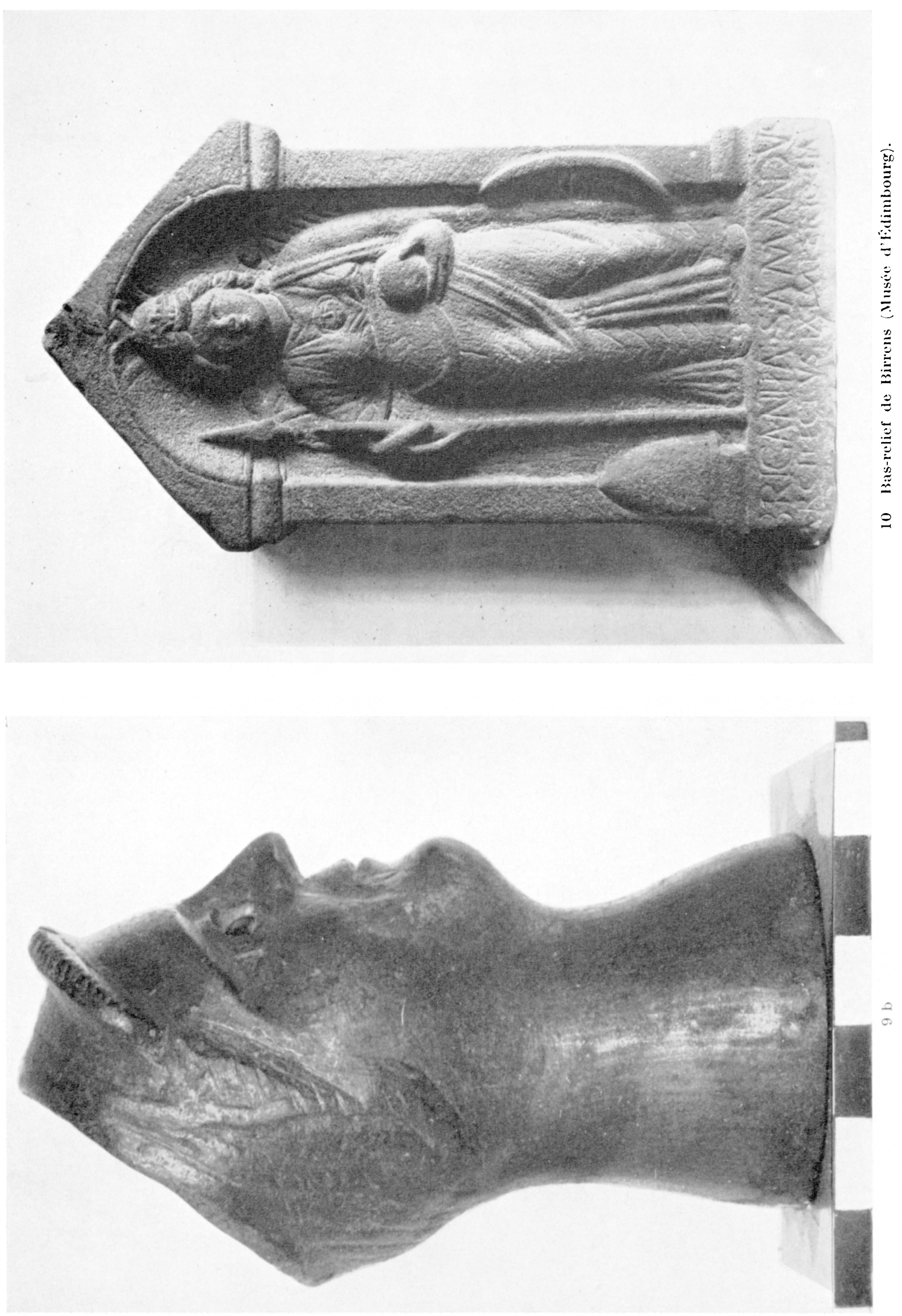
d'une couronne murale, les épaules supportent des ailes, la main droite tient un globe et une stèle omphalö̈de est posée pris de son pied galuche. Ces attributs complémentaires font de la Brigantia-Minerve de Birrens une figure syncrétique, qui associe la déesse romano-bretonne à la Victoire et à Juno Capleslis ${ }^{39}$. En liaison avec plusieurs inscriptions decouvertes dans le Yorkshire et pres du mur d'Hadrien ${ }^{40}$, ce relief date de la réorganisation sévérienne de la province de Bretagne. Plus réeent de deux siècles que la Minerve de I) inéault, il nous montre comment, à cette époque encore, une divinite indigene pouvait être revètue d'habits romains, sans pour cela perdre son nom.

Quant au style lui-mème, il est difficile de ne pas lui reconnaitre un aspect qui séerarte du canon classique. Bien que la recherche moderne ait tendance a réduire, sinon à nier, l'importance d'un style celtique de l'Indépendance dont on ne connait pas les cuvres aver certitudet1, on constate, a l'époque romaine, la coexistence de deux courants, l'un herlénisant et académique, l'autre populaire et réaliste. Ce dernier, qui a donné également les ex volo de bois des Soures de la Seine et de Chamalières, aurait porté en germe tout l'art du Moyen Age occidental. Preut-être y a-t-il, en effet, un air de famille entre la petite déesse inconnue de kerguilly en I)inéault et rertaines Vierges du xve siècle ${ }^{42}$.

René sANQTER.

39 X. Jolurre, Jea Brigantia, Dans Archaeological Journal, X(.11, 19.11, p. 36-61.

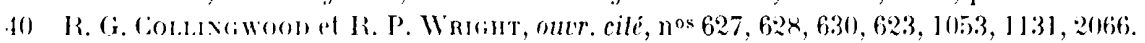

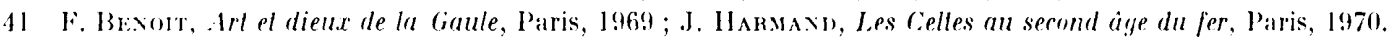

42 Je remereit de leuts conseils el de leus remarques, dont ils trouveront jei lecho, llme S. Boncher,

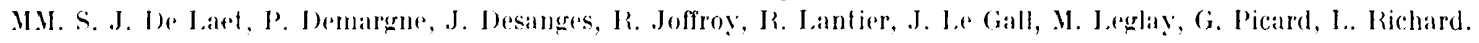
.V.I. F. Braemer. P.-.l. I uval, J. Harmand, ont bien voulu relire mon manuscrit : je leur "n suis tres reconnaissant. La carte est dur a .l. Goaster, dessinateur a leniversite de Bretagne Occidentale. I.es cliches 1 et 3 cont ete realisés

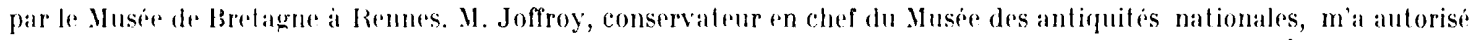

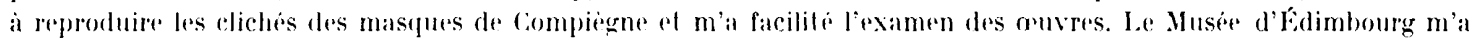
amablement communipue la plotographio du bas-pelief de Birrens, arec aulorisation de publication. M. J. Bourrhis, ingenienr chimiste an C.X.R.s., a realise Janalyse du melal dans le laboraloire de la Jjirection des antiquites pre-

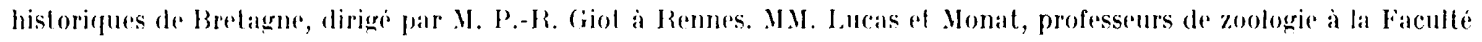
des Sciences de Brest, mont aide à identifier loisean. M. Mercier, benzier dart à Mohon Morbihan, ma initie aux techniques du bronze. 